\title{
Figures locales de l'« enfant de la rue ». Occupation de l'espace public au Sénégal et réification des catégories institutionnelles
}

\section{Safietou Diack}

\section{CpenEdition}

\section{Journals}

Édition électronique

URL : http://journals.openedition.org/anthropodev/821

DOI : 10.4000/anthropodev.821

ISSN : 2553-1719

Éditeur

Presses universitaires de Louvain

\section{Édition imprimée}

Date de publication : 1 décembre 2019

Pagination : 51-67

ISBN : 978-2-87558-940-8

ISSN : 2276-2019

\section{Référence électronique}

Safietou Diack, « Figures locales de l'« enfant de la rue ». Occupation de l'espace public au Sénégal et réification des catégories institutionnelles », Anthropologie \& développement [En ligne], 50 | 2019, mis en ligne le 19 décembre 2020, consulté le 25 janvier 2021. URL : http://journals.openedition.org/ anthropodev/821 ; DOI : https://doi.org/10.4000/anthropodev.821

La revue Anthropologie \& développement est mise à disposition selon les termes de la Licence Creative Commons Attribution 4.0 International. 


\title{
Figures locales de l'« enfant de la rue »
}

\author{
Occupation de l'espace public au Sénégal \\ et réification des catégories institutionnelles
}

Safietou Diack ${ }^{1}$

Au début des années 1990, le Sénégal ratifiait la Convention internationale relative aux droits de l'enfant (CIDE). Les enfants occupant les espaces publics urbains s'y définissent alors en problème social formulé notamment à travers la notion " enfants de la rue », et suscitent un intérêt croissant de la part des acteurs politiques et humanitaires. La CIDE impulse un renouveau des pratiques d'intervention sur ce phénomène avec l'entrée en scène de nouveaux acteurs. Dans le même temps elle charrie une représentation standardisée de l'enfance qui contribue à une approche partielle de la « problématique des enfants de la rue ". Ainsi l'action publique met en avant surtout les taalibe (enfants mendiant dans les rues) laissant en rade une catégorie comme celle du faqman (enfants et jeunes vivant dans la rue). Abordant cette dernière figure peu présente dans les études sur les « enfants de la rue » au Sénégal, cet article soulève les limites d'un traitement des catégories de la rue fondé sur des conceptions normatives de l'enfance et de sa socialisation.

In the early 1990s, Senegal ratified the International Convention on the Rights of the Child (CRC). Within this framework, children in public urban spaces are seen as a social issue as illustrated by the names they are given - "street children" - and they are objects of increasing interest from political and humanitarian actors. The Convention on the Rights of the Child has fostered a new form in related interventions and other actors have entered the scene. By promoting a standardized representation of childhood, the CRC only partially approaches this complex issue. Public action foremost focuses on taalibe (child beggars in the streets) and leave aside other children who nevertheless live on the streets, such as the fagman. By addressing them, this article explores the limits of an approach only based on normative conceptions of childhood and social integration process.

\footnotetext{
${ }^{1}$ Chercheuse post-doctorante, Institut de recherche pour le développement (IRD), Dakar, Sénégal ; s.diack@hotmail.fr
} 


\section{Introduction}

La production littéraire sur les " enfants de la rue " se développe à partir de la seconde moitié des années 1980. Elle contient alors pour l'essentiel des rapports d'ONG (Coloni, 1987) qui tirent la sonnette d'alarme et proposent des voies de réinsertion des enfants. Les travaux scientifiques émergent à peu près au même moment avec une prédominance des études anglo-saxonnes (Ennew, 1986 ; Aptekar, 1994). La recherche francophone sur le sujet prend forme quelques années plus tard avec des études menées sur des terrains africains (Combier, 1994). Au début des années 1990, on notera aussi les travaux de R. Lucchini (1993) et E. Taracena (1995) en Amérique latine. La réflexion sur les « enfants de la rue " s'ouvre sur un questionnement autour de ce concept. Qui sont les " enfants de la rue " ? Qui est-ce que cette expression désigne précisément ? L'objectif consistait à contrebalancer le "discours réducteur " ${ }^{2}$ de l'univers institutionnel pour modifier les représentations qui s'étaient formées sur l'« enfant de la rue ". Son association à l'espace de la rue en faisait une catégorie figurée par une image construite sur la compassion et/ou la méfiance. Pour rompre avec cette vision dichotomique, cette recherche entreprend de préciser l'objet en partant d'exemples observés sur le terrain. Ainsi dans une étude comparative menée dans trois villes d'Amérique latine (Montevideo, Mexico, Rio de Janeiro), R. Lucchini (1996) montrait en quoi l'enfant de Montevideo et celui de Rio de Janeiro ne pouvaient être regroupés dans une même définition de l' " enfant de la rue » malgré certaines convergences. Des critères comme la place de l'enfant dans sa famille, la configuration de l'espace de la rue, spécifiques dans chaque contexte, suggéraient des distinctions. Ces travaux empiriques ont permis de mettre au jour le caractère hétérogène de la catégorie « enfant de la rue ».

Au cours de ma thèse, lorsque j'évoquais mon travail sur les " enfants de la rue " au Sénégal, les commentaires qui fusaient renvoyaient systématiquement aux enfants taalibe mendiant dans les rues. La prégnance de cette catégorie dans la perception locale des " enfants de la rue » vient certainement de la grande visibilité de cette mendicité infantile dans l'espace public. Dans les villes sénégalaises, des enfants munis de leur sébile, supposés confiés à un marabout-enseignant pour recevoir un enseignement et une éducation religieuse, mendient à tous les coins de rue. De ce fait lorsqu'on pose la " problématique des enfants de la rue ", les débats tournent essentiellement autour de la mendicité et des maltraitances que connaissent certains de ces enfants. Médias et ONG ont grandement participé à la problématisation de ce phénomène en dénonçant les conditions de vie et les violences que connaissent ces enfants. Leurs voix imposent la réalité de ce problème et suggèrent une action urgente. Cependant sa mise en avant empêche d'aborder la question de la présence d'enfants et de jeunes dans les espaces publics urbains sénégalais dans sa complexité et sa diversité. En effet à côté des taalibe, on distingue (parmi d'autres catégories que nous n'aborderons pas ici) les faqman,

\footnotetext{
${ }^{2}$ Je reprends ici une expression de l'article de R. Lucchini (1998), " L'enfant de la rue : réalité complexe et
} discours réducteur $"$. 
catégorie d'enfants et de jeunes relativement invisible et absente des débats publics, assimilée autant par les populations que les autorités à une délinquance juvénile.

Comme l'a souligné E. Taracena (1995: 102), « [...] il est important de dépasser la tendance à présenter une image unifiée des enfants des rues [...] » pour une meilleure compréhension des enjeux sociaux (et politiques) qui entourent ce phénomène. Dans cette optique cet article analyse la manière dont la présentation de la «problématique des enfants de la rue " au Sénégal en termes de vulnérabilités (taalibe) mais aussi d'inadaptation sociale (faqman) participe à une perception réductrice des usages juvéniles des espaces publics alors vus comme des situations d'exploitation des enfants, ou comme l'expression d'une déchéance physique et morale. II montre dans un premier temps la façon dont les mesures politiques concernant les " enfants de la rue », depuis le début des années 1990, dans le contexte de l'adoption de la Convention internationale relative aux droits de l'enfant (CIDE), suggèrent une appréhension de la problématique essentiellement centrée sur la mendicité d'enfants taalibe et les corollaires de cette pratique définie comme une entreprise d'exploitation. Dans un second temps il présente comment la perception négative des faqman oriente une volonté de normaliser cette catégorie par des pratiques d'intervention qui tentent de les replacer dans des itinéraires sociaux formels, quand bien même la diversité des profils qu'elle renferme montre que la présence dans la rue de ces jeunes n'augure pas une rupture sociale systématique.

Il est difficile de situer avec précision les périodes auxquelles ces deux figures, faqman et taalibe, apparaissent effectivement. Les taalibe sont liés à l'existence des daara, espaces d'enseignement coranique et de socialisation séculaires qui se développent avec l'arrivée de l'islam dans cette partie de l'Afrique de l'Ouest vers le XI ${ }^{\mathrm{e}}$ siècle. Quant aux faqman, leur présence dans la rue est corrélée à la crise économique et aux effets des ajustements structurels des années 1980 (Faye et Thioub, 2003). Il se peut que leur existence remonte à bien plus loin mais l'absence de données suffisantes ne nous permet pas de confirmer cette hypothèse ${ }^{3}$. Ces deux catégories de la rue occupent donc le paysage urbain sénégalais depuis de nombreuses années. Mais leur construction en tant que problème social formulé à travers l'expression " enfants de la rue " commence dans les années 1990 lorsque les premières ONG qui interviennent aujourd'hui encore auprès des " enfants de la rue " arrivent sur la scène locale (Enda jeunesse-action en 1985, Avenir de l'enfant en 1991, Village pilote en 1993, etc.), pour ensuite se multiplier dans le courant des années 2000 (Samu social Sénégal, centre Nazareth, Solidarité pour les enfants de la rue, etc.). L'adoption de la CIDE, ratifiée par le Sénégal en 1990, mobilise autour des « enfants de la rue " et renouvelle les pratiques d'intervention, avec l'implication de ces nouveaux acteurs. En effet, jusque-là, la prise en charge des occupations juvéniles des espaces publics s'intégrait dans une politique de gestion des marges urbaines oscillant entre répression, régulation et réglementation. Cette politique consistait à retirer de l'espace public tous ceux qui en perturbaient l'ordre, en les enfermant ou les repoussant vers les

\footnotetext{
${ }^{3}$ La catégorie du faqman était jusqu'ici relativement méconnue, exceptées l'étude de Dramé (2010)
} réalisée pour le compte du Samu social Sénégal et la thèse que nous y avons consacrée (Diack, 2017). 
périphéries urbaines (Collignon, 1984 ; Diop, 1990 ; Faye et Thioub, 2003). Si elle introduit une approche d'aide et d'accompagnement, la CIDE comme référentiel de base charrie aussi une représentation basée sur une conception de l'enfance qui s'est développée dans le monde occidental et qui tend à s'imposer en norme universelle. Elle fonde une perception normative de l' "enfant de la rue » qui occulte la réalité vécue de cette catégorie, pour une réalité représentée, ainsi que nous le verrons dans la troisième partie qui propose de considérer les terminologies locales pour mieux appréhender les catégories de la rue et leur réalité empirique.

Cet article repose sur une enquête de terrain réalisée à Dakar entre 2013 et 2015 dans le cadre de mes recherches doctorales. Les données mobilisées ont été recueillies à partir d'une observation participante effectuée au centre Nazareth, une structure qui mène un " projet de réinsertion des enfants en rupture familiale » depuis 2004. Des entretiens avec des faqman, des anciens faqman et des acteurs de l'intervention (travailleurs sociaux, éducateurs, animateurs de rue, responsables d'ONG, etc.) complètent des observations menées sur les lieux de vie des faqman dans la rue.

\title{
Une mobilisation politique et institutionnelle autour de la catégorie du taalibe-mendiant
}

La mendicité infantile est courante dans la capitale sénégalaise. Tous les jours, on peut observer des enfants seuls, en groupe, ou en compagnie d'adultes, mendier au coin d'une rue, devant les commerces... Parmi ces enfants mendiants, certains davantage que d'autres retiennent l'attention des médias et des acteurs politiques et humanitaires, les enfants localement désignés par le terme taalibe. Ces enfants dont on présume qu'ils sont confiés à un marabout-enseignant, ou sëriñ daara en wolof ${ }^{4}$, chargé de leur enseigner le Coran et la pratique religieuse, sont soumis à de longues heures de mendicité. Ils doivent quotidiennement remettre à leur sëriñ daara une somme d'argent fixée par ce dernier, ainsi que des denrées (riz, sucre, bougies, etc.). Lorsque le montant demandé n'est pas versé ces enfants risqueraient des punitions dont la violence conduit les ONG à employer un terme aussi fort que celui d' « esclavage " pour qualifier leur situation :

\begin{abstract}
Leurs professeurs (marabouts), qui font office de tuteurs de facto, les soumettent à des formes souvent extrêmes de maltraitance, de négligence et d'exploitation. [...] De nombreux marabouts obligent les enfants, appelés talibés, à mendier dans les rues pendant de longues heures - pratique constitutive de la pire forme de travail des enfants selon la définition employée par l'Organisation internationale du travail (OIT) et les soumettent à des violences physiques et psychologiques souvent brutales. (Human Rights Watch, $2010: 6$ )
\end{abstract}

Identifié comme "problématique des enfants de la rue », le phénomène relèverait d'une " présence massive des enfants dans la rue à des fins d'exploitation " d'après un rapport de l'État sénégalais rendant compte des résultats du programme de " retrait des

\footnotetext{
${ }^{4}$ Le wolof est la principale langue véhiculaire au Sénégal.
} 
enfants de la rue » initié en juin $2016^{5}$. Les documents institutionnels signalent l'ampleur du phénomène et mettent l'accent sur des violences et maltraitances dont les médias se font régulièrement l'écho. Parmi les épisodes les plus dramatiques relayés par la presse, le décès de près d'une dizaine de taalibe dans l'incendie de leur daara ${ }^{6}$ en mars 2013. En se saisissant de ces faits-divers, la presse rappelle régulièrement à l'opinion publique la condition de ces enfants mendiants et participe à la problématisation du phénomène.

Avec l'adoption de la CIDE, les taalibe deviennent une priorité pour les programmes internationaux en faveur de l'enfance au Sénégal. Les premières interventions menées en partenariat avec I'UNICEF visent à les placer dans de meilleures conditions d'apprentissage. Par la suite l'idée d'intégrer les daara au système éducatif formel intervient avec le lancement en 2002 d'un programme de "modernisation des daara" (Hugon, 2015). De nombreuses familles, particulièrement dans les zones rurales, privilégient, et ce pour diverses raisons, ce modèle éducatif pour leurs enfants (Chehami, 2016). La formalisation des daara intégrerait ces enfants aux statistiques officielles et participerait ainsi à l'atteinte de l'objectif d'une éducation pour tous. Des enjeux politiques sous-tendent donc ces initiatives et expliquent en partie l'attention portée sur les taalibe-mendiants comme catégorie de la rue.

Le Sénégal dispose d'un appareil institutionnel particulièrement élaboré pour prendre en charge les problèmes de l'enfance ${ }^{7}$ sur la base $d^{\prime}$ 'une approche holistique. Cependant il persiste une gestion compartimentée relevant d'une approche en termes de droits et de protection, au détriment d'une approche compréhensive ainsi que le proposent les travaux de J. Chehami (2013) et K. Sané (2017) qui éclairent les mécanismes sociaux relatifs notamment à la place du don et de l'aumône dans la société sénégalaise, qui participeraient à la "pérennisation» de la mendicité d'enfants taalibe. Ces analyses permettent par ailleurs de sortir d'un amalgame possible entre cette catégorie des taalibe-mendiants apparue en milieu urbain à la faveur de crises socio-économiques et d'un exode rural massif vers les années 1970, et le taalibe en tant qu'apprenant confié dans l'optique d'une éducation religieuse et spirituelle. En effet il faut préciser que le terme taalibe possède à l'origine deux sens. II désigne dans un premier sens l'élève du daara. Dans un second sens il se rapporte aux disciples d'une confrérie religieuse qui font allégeance à un sëriñ ou marabout, dont ils deviennent les taalibe, les disciples (Diop, 2012). Le taalibe confié au daara doit y recevoir un enseignement et une éducation basés sur des préceptes islamiques. En wolof on le désigne comme ndongo daara (élève du daara) ou njàngaan (apprenant) du verbe jàng signifiant apprendre, étudier.

À côté de l'État, les ONG et les organismes internationaux se mobilisent aussi autour des enfants taalibe. Les chiffres qu'ils présentent à l'appui de leurs plaidoyers soulignent une envergure du phénomène dans l'espace public au Sénégal. L'ONG Human Rights Watch,

\footnotetext{
${ }^{5}$ République du Sénégal, Initiative nationale pour le retrait des enfants de la rue, mars 2017.

6 Source: https://www.jeuneafrique.com/depeches/38919/politique/senegal-neuf-enfants-morts-danslincendie-dune-ecole-coranique/ (consulté le 15/10/2019).

${ }^{7}$ République du Sénégal, Stratégie nationale de protection de l’enfant, décembre 2013.
} 
qui a publié plusieurs rapports sur le sujet ${ }^{8}$, estime qu'«au moins 50000 enfants fréquentant des centaines d'internats coraniques (daaras) au Sénégal sont soumis à des conditions qui s'apparentent à de l'esclavage » (Human Rights Watch, $2010: 6$ ). Dans un rapport publié en novembre 2007, I'UNICEF estimait à 7600 le nombre d'enfants mendiants dans la région de Dakar dont $90 \%$ seraient des taalibe. Ces chiffres ont davantage vocation à interpeller l'opinion publique sur la situation de ces enfants mendiants qu'à exprimer une réalité tangible. En effet, la proportion de taalibe (élèves de daara) qui se retrouvent à mendier sous la contrainte est difficile à quantifier en raison d'une difficulté à les distinguer d'autres catégories d'enfants mendiants. Leur identification au-delà d'une perception ordinaire qu'on peut en avoir en sillonnant les rues dakaroises (ou celles d'autres villes sénégalaises) n'est pas si simple.

La volonté politique de retirer les taalibe-mendiants de la rue, alliée à la mobilisation des acteurs humanitaires, locaux comme internationaux, montre tout l'intérêt porté à cette jeunesse qui investit les espaces urbains, intérêt cependant focalisé sur une catégorie d'enfants mendiants. Cette concentration altère une appréhension globale et une compréhension réelle des formes d'occupations et d'usages juvéniles des espaces publics urbains. Les faqman constituent une autre figure de la rue, qui mobilise certes l'action d'ONG locales (nous en citions quelques-unes en introduction), mais peine à se départir d'une perception sociale négative, qui se reflète dans le traitement tant par les acteurs humanitaires que les autorités publiques.

\section{Perception collective et ambivalences dans le traitement de la figure locale du faqman}

Les faqman sont une catégorie d'enfants et de jeunes qui ont quitté leur famille, leur daara, ou un lieu d'apprentissage, et qui vivent dans la rue. En 2013, la télévision sénégalaise rediffusait un téléfilm intitulé Faxxman, réalisé dans les années 1990. Cette fiction retrace le parcours d'un adolescent vivant dans un quartier populaire de Dakar avec sa famille dont le chef, polygame et au chômage, peine à subvenir aux besoins de sa nombreuse progéniture. Une altercation avec celui-ci conduit l'adolescent à quitter le domicile familial. Après quelques jours d'errance, il rejoint une bande de faqman. Retranché dans un édifice abandonné, ce groupe est dirigé par un chef sous l'influence constante des stupéfiants qu'il ingère. II arrive néanmoins à organiser le groupe qui vit essentiellement de délits. Les personnages de ce téléfilm sont présentés en totale rupture avec leurs familles, complètement en marge et dans le déni des repères et principes sociaux normatifs, recréant une communauté déviante avec ses propres règles. Cette fiction présente une vision populaire du faqman comme marginal dont le quotidien est rythmé par la violence et les délits. Le parcours de Momar ${ }^{9}$, ancien faqman, rejoint le portrait qui est fait du « jeune de la rue » dans le téléfilm Faxxman :

\footnotetext{
${ }^{8}$ Human Rights Watch, 2010, 2014 et 2017.

${ }^{9}$ Les prénoms des jeunes sont modifiés.
} 


\begin{abstract}
Moi je viens d'un daara, c'est mon père qui m'y avait conduit et je me suis enfui pour revenir à la maison. Quand je suis revenu, on m'a ramené au daara et je me suis enfui à nouveau. On $\mathrm{m}^{\prime}$ a enchaîné $\left[j e ̀ n g^{10}\right]$ et par la suite on $\mathrm{m}^{\prime} \mathrm{a}$ emmené à Kokki ${ }^{11}$. C'est à ce moment-là que j'ai fugué, je suis venu à Dakar là où y a les tubaab [il parle du centreville], mais quand j'étais avec ces tubaab là-bas je ne volais pas, je mendiais. Mais les boys [les autres faqman] que je fréquentais ils volaient et avaient des millions. Un jour j'ai volé le sac d'un tubaab, nous étions assis comme ça à discuter j'ai volé son sac et il y avait 800000 [francs CFA] dedans. J'étais donc dans le milieu ${ }^{12}$, mais Dieu m'a aidé parce que je ne prenais pas de ginz ! Je fumais de la cigarette, mais je ne fumais pas de boon [chanvre]. Moi notre seigneur m'a aidé, mais personne n'était aussi mauvais que moi ! Tu disais ou faisais des business même et je te plantais [il fait mine de poignarder quelqu'un]. Est-ce-que tu m'entends bien ? Je suis resté dans le milieu, j'ai fait des allers-retours, tu vois, [...] j'ai signé 14 mandats de dépôts, j'ai fait la prison 14 fois [...]. J'en ai entraîné beaucoup sur cette voie parce qu'ils voyaient Geste-bi [c'est son surnom] avec de l'argent, ils n'ont jamais su ce que c'était que d'être bandit ou ce genre de choses, Geste-bi leur donnait un peu d'argent, ou bien quand Geste-bi allait quelque part, ils le suivaient et regardaient ce que Geste-bi faisait. J'avais dans les 500 000, des millions, l'argent j'arrivais à l'avoir comme je voulais et j'ai influencé beaucoup de boys. Maintenant je m'en suis sorti, j'ai arrêté, pourtant je disais que seule la mort pouvait me faire arrêter [...] ! (Momar, 30 ans, Dakar, janvier 2014)
\end{abstract}

Le récit de Momar, qui a vécu une dizaine d'années dans la rue, présente les pratiques et comportements qui se rapportent typiquement au faqman. On est entre rupture, après plusieurs fugues dont la dernière le conduit dans les rues du centre-ville dakarois; délinquance, lorsqu'il passe de la mendicité au vol; et violence lorsqu'il parle de sa promptitude à user d'armes blanches. Momar évoque aussi le ginz qui consiste en l'inhalation de diluant cellulosique pour se droguer. II constitue une pratique courante mais non généralisée chez les faqman. Certains jeunes après un usage temporaire décident d'arrêter, selon eux en raison de ses effets nocifs sur la santé et de l'image de soi négative qu'il renvoie :

[...] Quand tu le fais on ne te considère pas. On ne te respecte pas. Si tu avais l'intention de venir vers moi, comme aujourd'hui tu viens, comme je suis à Sandaga [un des milieux des faqman dans un marché du centre-ville dakarois], tu me trouves là-bas, assis, cool quoi, bien, je ne fais rien de mal. Tu reviens, et je suis la raison de toutes tes visites, tu viens exprès pour discuter avec moi. Un autre jour tu reviens et tu trouves que j'ai pris du ginz et que je suis saoul, je te demande alors, si tu me vois comme ça, dans cet état, est-ce que tu seras encore motivée ? (Ali, 15 ans, Dakar, février 2014)

Cette conscience de l'image de soi négative, que renvoie l'usage de substances psychotropes, nuance une représentation du faqman comme individu en rupture avec les normes et conventions sociales. Au regard du caractère évolutif et dynamique de l'expérience de la rue, cette image du faqman doit être complétée. Comme réalité

\footnotetext{
${ }^{10}$ Pratique consistant à attacher les pieds et mains afin que le taalibe ne fugue pas à nouveau.

${ }^{11}$ Un des plus anciens centres d'enseignement coranique situé dans le village de Kokki rattaché à la région de Louga au nord-ouest du Sénégal.

${ }^{12}$ Les faqman utilisent ce terme pour parler de leur expérience de la rue, ils diront par exemple " je suis dans le milieu depuis... ", " ma vie dans le milieu... ", " quand je suis arrivé dans le milieu... ", plutôt que d'utiliser le terme rue qui se dit mbedd en wolof. Ils l'utilisent également pour désigner les lieux où ils vivent et se retrouvent.
} 
empirique la catégorie faqman se décline en profils distincts et ne se limite pas à l'image du déviant. Le parcours qui fait sa marginalité est moins linéaire qu'il n'y paraît. L'image du marginal, du délinquant, correspond davantage à une étape dans sa " carrière de rue " (Lucchini, 2001) qu'elle ne résume son parcours. La catégorie observée sur le terrain présente divers profils dont certains s'éloignent des comportements marginaux qui participent à la définition sociale courante du faqman. Momar incarne la figure du faqman ancien taalibe, un des trois profils que j'observe dans les rues de Dakar sur la base des raisons que ces jeunes invoquent quant à leur présence dans la rue. Après leur fugue du daara, certains de ces enfants trouvent refuge dans les milieux faqman où ils se cachent de leur famille ou de leur sëriñ daara qui parfois se mettent à leur recherche. D'autres au contraire, d'emblée ou dans le cours de leur expérience de la rue, évitent ces milieux qui ont pour eux mauvaise réputation et sont la source d'une influence néfaste :

\begin{abstract}
Je sais que si tu y restes trop longtemps tu peux attraper des maladies ou t'habituer au ginz, ou à la cigarette et ne plus pouvoir t'en sortir, et ça, ça peut te nuire. C'est que, avant je faisais tout, je faisais le ginz, je fumais, mais à un moment je me suis rendu compte que ça n'avait pas d'utilité, ça ne menait nulle part, j'ai alors arrêté de fréquenter ces boys là pour rester à la salle de jeux pour ne pas faire ces choses. (Demba, 15 ans, Dakar, février 2014)
\end{abstract}

Demba a vécu environ cinq ans dans la rue. Son parcours illustre l'ambivalence du rapport de l'enfant à la rue et son caractère évolutif déjà évoqué par R. Lucchini (ibid.) à travers la notion de "carrière " pour souligner les diverses étapes que comporte cette expérience. Demba décrit un début de parcours marqué par l'adhésion aux pratiques qui font la perception péjorative du faqman et dont il fait l'apprentissage dans les milieux. II parle ensuite d'une introspection qui lui aurait permis de prendre conscience du caractère " inutile » de ces pratiques. La crainte d'être renvoyés au daara s'ils rentraient, en plus de l'envie de profiter d'une liberté nouvelle, poussent ces enfants à prolonger leur présence dans la rue. S'ils continuent d'y dormir le soir venu, ils privilégient en journée des espaces plus conventionnels comme les salles de jeux ou des centres d'accueil de jour ouverts par des structures caritatives... En effet, les milieux où se retrouvent habituellement les faqman sont souvent caractérisés par une insalubrité qui les expose à des affections diverses, et/ou par une forte consommation et vente du diluant cellulosique servant au ginz. Autant de contraintes et de tentations que certains enfants vivant dans la rue cherchent à éviter.

Comme second profil, nous avons le faqman ancien travailleur migrant. II a quitté sa famille pour trouver du travail en ville et aider celle-ci. Pour ce jeune il est plus juste de parler de départ que de fugue, quand bien même la famille n'est souvent pas au courant de son projet de partir. Contrairement au premier profil il conserve un contact, même sporadique, avec ses proches auprès desquels il retourne périodiquement après avoir pu faire quelques économies. A. Guillou (2009) fait des observations similaires au Cambodge auprès d'enfants dont la présence dans la rue est due à la faiblesse des revenus de leurs familles. Ces enfants expliquent qu'ils retournent chez eux à chaque fois qu'ils ont un peu $d^{\prime}$ argent pour aider leurs parents. C'est un profil qui résiste à une définition de l'enfant qui vit dans la rue en termes de rupture des liens familiaux et de délinquance. Arrivé à Dakar, 
il exerce généralement des petits boulots dans le secteur économique dit informel. II s'oppose à une représentation de l'enfant dépendant, présente dans la CIDE et que l'État sénégalais et les organismes d'intervention relaient. Pour ces institutions, la prise en charge de l'enfant est en effet du ressort des parents ou tuteurs. Or, dans la pratique locale, bien avant sa majorité l'enfant peut être en quête d'autonomie et vouloir soutenir sa famille. Cette volonté de contribuer à l'économie de la famille est nourrie dès le plus jeune âge à travers une socialisation qui inclut l'attribution de tâches et de responsabilités aux enfants. D. Thorsen et M. Jacquemin (2015), à partir des trajectoires d'enfants travailleurs migrants en Côte d'Ivoire et au Burkina Faso, ont montré la façon dont les catégories institutionnelles élaborées autour des circulations enfantines réduisent ces enfants en objets à protéger et à secourir ; les auteures soulignent par ailleurs qu'en milieu rural les enfants, en participant aux travaux des champs, aux travaux ménagers, aux soins des plus jeunes, etc., s'initient progressivement au travail et à une forme d'autonomie. Ce jeune qui entreprend d'aller vers la capitale pour trouver un travail se sent des obligations, une responsabilité envers ses proches. La rue constitue alors à la fois un espace de vie et de travail. Bien entendu il n'est pas à l'abri de violences multiformes et de diversions susceptibles de prendre l'avantage sur les motivations affectives et économiques à l'origine de sa présence dans la rue. Mais, dans des contextes où les équilibres économiques et sociaux qui doivent favoriser l'accomplissement et l'intégration des individus sont fragiles, la dimension d'apprentissage et d'opportunités que renferment certaines trajectoires perçues comme marginales ou déviantes ne peut être occultée.

Le dernier profil est celui du faqman qui fugue du cadre familial. II quitte son domicile sous l'influence de rencontres, de l'environnement, ou du fait de conflits familiaux. Attiré par la proximité des milieux faqman du domicile, il y expérimente la prise de drogues (chanvre, ginz), avant de s'installer durablement dans une marginalité qui crée sa stigmatisation et son exclusion. Ce profil semble être à l'origine du terme même de faqman et de la perception négative qui lui est rattachée. Faqman est une association du wolof faq et de l'anglais man. Appliqué aux jeunes vivant dans la rue, faq se traduit communément par fuguer. Cependant le terme signifie aussi arracher, briser, évoquant ainsi une rupture, souvent violente, qui se produit entre une entité et un ensemble dont elle est normalement une partie intégrante et dépendante. L'entité s'épanouissant grâce au lien avec cet ensemble, la rupture engendrerait une dégénérescence, d'ordre moral principalement dans le cas des faqman.

L'image du faqman comme délinquant présente l'inconvénient de le cantonner à une partie de ses pratiques dans la rue (vol, usage de drogues, pratiques homosexuelles, etc.), pour lui façonner une identité déviante qu'on retrouve dans les discours des populations à son sujet. En attestent les propos d'un loueur de vélo travaillant à proximité d'un " point de chute ${ }^{13}$ situé sur un parking accueillant des cars de transport interrégionaux à Dakar :

\footnotetext{
${ }^{13}$ Les intervenants sociaux désignent ainsi les endroits où des faqman se retrouvent.
} 
Ils ne sont pas faciles à aider. On leur a permis de dormir dans les cars mais ils font toutes sortes de choses dedans, ils s'y soulagent [parce qu'il retrouve des excréments dans les cars usagés aussi stationnés sur place] et font des pratiques homosexuelles. Ils dégradent les cars. Ils brisent les vitres et volent la ferraille et le bronze pour les revendre. Leur seule préoccupation c'est de se faire de l'argent. (extrait de carnet de terrain, Dakar, janvier 2014)

Ces griefs exprimés à l'égard des faqman sont des arguments que l'on retrouve aussi dans l'univers de l'intervention, avec des ONG locales qui travaillent à leur « retrait de la rue » et à leur " réinsertion ». Ainsi le centre Nazareth effectue deux fois par semaine des " actions-rue » à Dakar. Ces visites dans les milieux des faqman inscrites dans le cadre d'un " travail social de rue " servent à créer avec ces jeunes une relation de confiance dont la finalité est la sortie de la rue et le retour en famille, sinon l'intégration dans un centre d'hébergement. Le centre Nazareth propose également des accueils hebdomadaires dans ses locaux où des services (soins médicaux, linge, repas) et activités diverses (discussions de sensibilisation, jeux de société, etc.) leur sont proposés. Si cette démarche de "réinsertion " vise l'ensemble des faqman comme enfants et jeunes vivant dans la rue, une forme de sélection s'opère dans la prise en charge concrète. Le faqman qui souhaite bénéficier de cet appui doit montrer les signes d'une volonté de rompre avec les habitudes acquises dans la rue. Ainsi les jeunes qui présentent une addiction au ginz, s'ils ne sont pas totalement exclus des programmes, sont tout de même abordés avec quelques réserves, de même que ceux qui ont passé de nombreuses années dans la rue. Les intervenants considèrent que la réalisation de leur sortie de la rue sera plus ardue en raison du temps et des habitudes acquises dans la rue, en contradiction avec les règles sociales.

La représentation négative du faqman participe à créer chez les intervenants sociaux un sentiment ambivalent vis-à-vis de cette catégorie. Un sentiment qui oscille entre méfiance et compassion. La dépendance au ginz d'un jeune influencera la décision d'une structure de lui apporter un soutien immédiat ou de le tenir dans une phase d'observation qui consiste simplement à garder un contact à travers les " actions-rue ». Pour exemple, lors d'une réunion du centre Nazareth, les intervenants discutent le cas d'un jeune prénommé Nuur. Âgé de 19 ans, sa présence dans la rue n'est pas encore permanente mais il fréquente régulièrement des milieux faqman qui se trouvent à proximité de son domicile. Voyant qu'il vit encore avec sa famille, pour que sa présence dans la rue ne devienne pas définitive, les intervenants de Nazareth décident de lui apporter une aide. L'équipe évoque plusieurs propositions. Celle qui trouve le plus d'écho est de lui trouver un emploi dans une des écoles du réseau de la structure. Mais des objections sont émises en raison de sa pratique du ginz : "Est-il réellement stable ? Et s'il se mettait à commettre des vols sur son lieu de travail ? Peut-on prendre un tel risque ? Comment se justifiera-t-on s'il brise la confiance placée en lui ? ". Sa situation actuelle ne permet pas un tel pari : " II faut qu'il se stabilise davantage ! "; " Mais il ne peut pas rester à la maison sans rien faire ! Ça pourrait le faire replonger ! » disent les animateurs.

Ces intervenants ont des positions divergentes. Certains pensent qu'il faut prendre le risque de faire confiance au jeune homme. Les autres recommandent la prudence, 
doutant de la fiabilité du garçon. Ils souhaitent intervenir en l'aidant à trouver un emploi pour que sa fréquentation des milieux ne devienne pas permanente mais ne veulent pas prendre le risque de le voir briser la confiance qu'ils placeraient en lui. Finalement la décision qui est prise est de continuer à observer l'évolution de Nuur en gardant le contact avec lui afin de voir s'il respecte l'engagement pris d'arrêter le ginz.

Si l'attitude des acteurs humanitaires à l'égard des faqman dénote une certaine ambivalence, celle des autorités sanctionne clairement la présence de ces jeunes dans l'espace public. La police opère ainsi des interventions régulières dans leurs milieux :

Quand nous arrivons sur le "point de chute ", les jeunes ont déménagé de leur coin habituel. Dakar s'est rafraîchie. Alors cherchant la chaleur du soleil, ils ont quitté l'ombre de l'immeuble au pied duquel ils se réunissaient, pour s'installer au centre du terrain vague, plus ensoleillé. Certains ont leur chiffon imbibé de diluant, d'autres roulent un joint. Deux jeunes organisent une partie de dames. [...] L'ambiance est alors détendue quand au loin apparaît un pick-up blanc qui progresse vers le groupe. C'est la police. Le véhicule à peine repéré, il s'ensuit une débandade qui vide le lieu de ses occupants. Sauf M., un faqman d'une vingtaine d'années qui lui ne bouge pas. Pourquoi ne prend-il pas la fuite comme ses camarades? Le véhicule qui ne poursuit pas les fuyards s'arrête à notre hauteur. Deux agents en civils en descendent. Le premier, un homme grand et mince, âgé d'une trentaine d'années. Le second visiblement plus âgé, la cinquantaine peut-être, grand et légèrement bedonnant, s'adresse à nous [animateurs du centre d'accueil et chercheur]. II demande ce que nous faisions sur les lieux, " avec ces jeunes ». Les animateurs de Nazareth expliquent:

"Nous sommes un centre et notre travail consiste à sensibiliser ces jeunes afin qu'ils retournent chez eux.

- Mais vous ne pouvez pas venir les trouver ici quand même! Si vous voulez, nous on vous les amène ! » objecte l'agent.

Leurs interventions visent à décourager les jeunes de rester dans la rue et de se fixer sur ces lieux, chose que l'intervention du centre paraît favoriser selon lui. Sans doute est-ce la raison pour laquelle ils ne les ont pas poursuivis, se contenant juste de les faire fuir. II poursuit :

"Ils restent là à ginzer et à fumer, et après ils vont voler. C'est tout ce qu'ils savent faire.

— Et toi ? Qu'est-ce que tu fais ici ? Enchaîne-t-il s'adressant à M.

- Je suis avec eux [les agents du centre]. Pour leur montrer les boys [les autres faqman]. "

Malheureusement, son apparence le dessert. II paraît saoul et son sweet-shirt blanc a depuis longtemps perdu sa couleur d'origine. Le mensonge ne passe pas. Peut-être comptait-il sur l'équipe de Nazareth pour l'appuyer ? Ce que celle-ci ne fait pas.

« Mais tu es saoul toi ! I'interrompt l'agent. Le jeune homme nie.

- C'est pas vrai ! Tu es saoul ! insiste le policier, ramassant à ses pieds un flacon à moitié rempli de diluant.

- Embarque-le [...] ! ordonne-t-il à son jeune collègue, jusque-là silencieux, se contentant d'observer la scène.

- Ne me touche pas! » ose M. à l'intention de l'agent qui l'empoigne. Le garçon ne se débat pas malgré sa résistance verbale. Mais cette objection lui vaut une gifle retentissante avant qu'il ne soit embarqué à l'arrière du pick-up qui s'éloigne avec sa prise [...]. (extrait carnet de terrain, Dakar, février 2014)

Cet épisode montre la prédominance d'une approche répressive dans la gestion des faqman par les autorités. Les agents de police considèrent que ces interventions peuvent 
avoir un effet dissuasif et les inciter à regagner leurs domiciles. Mais les jeunes ne font que se déplacer d'un point à l'autre, selon la régularité des interventions policières.

Le traitement des " enfants de la rue " au Sénégal suggère une appréhension de ce fait social comme problème touchant des enfants confrontés à des contraintes économiques et sociales qui les poussent à des états de vulnérabilité et les confrontent à des situations de maltraitance, d'exploitation et de perdition morale. Couvrant une tranche d'âge relativement étendue (les faqman que je rencontre à Dakar sont âgés de 9 à 10 ans pour les plus jeunes et de près de 30 ans pour les plus âgés), réunissant des anciens taalibe fuguant de leur daara, des jeunes quittant leurs familles en raison de maltraitances, de conflits, ou dans l'optique d'une vie meilleure, la figure du faqman donne à voir la diversité des trajectoires qui mènent à une présence plus ou moins prolongée dans la rue. Elle montre surtout que face aux contraintes ces jeunes n'ont pas pour seule réaction la fuite vers la rue. Celle-ci n'est pas toujours abordée dans une logique de rupture sociale. Elle est également envisagée comme ressource et comme espace de rencontres et d'opportunités dans le cadre d'une quête de reconnaissance sociale, ainsi que le suggèrent aussi les travaux de $C$. Dugrand (2013) sur les Shégués à Kinshasa. Cependant cette conception de l'expérience de la rue ne peut être admise qu'en dehors de représentations normatives selon lesquelles les socialisations juvéniles doivent se dérouler en des lieux formels.

\section{Considérer les terminologies locales pour dépasser une vision monolithique}

D. Stoeklin (2000) a observé en Chine à partir des profils de l'« enfant-mendiant » et de l'« enfant-vagabond " les différences de perception et de traitement existant entre les catégories de la rue selon leur degré de correspondance avec une représentation normative de l'enfant, qu'elle soit culturellement construite, ou institutionnellement comme la catégorie " enfant de la rue ". II remarque que l'enfant-mendiant pratiquant cette activité sous la tutelle des adultes fait partie d'un système hiérarchisé où il figure au bas de l'échelle, ce qui limite sa sociabilité dans la rue. L'enfant-vagabond comme le faqman, à travers les différentes pratiques de survie dont il fait l'apprentissage dans la rue, dispose d'une plus grande autonomie. Il développe des compétences dans la rue mais pour satisfaire ses propres besoins. Par sa position, plus que l'enfant-mendiant, il va à l'encontre des représentations culturelles de l'enfant dans la société chinoise. II est donc davantage marginal. L'enfant-vagabond s'émancipe des contraintes sociales alors que l'enfant-mendiant reste une composante d'un réseau hiérarchisé. II y a un décalage, nous dit D. Stoecklin (ibid.), entre l'image idéale de l'enfant et la réalité, qui peut être déterminant dans la nature et le degré de violence des dispositions que les autorités prennent pour réduire cet écart. Au Sénégal, le taalibe-mendiant encore associé à l'institution du daara malgré des temps de présence dans la rue reste dans une conception normative de l'enfant en gardant cette attache avec un cadre social formel. Le faqman en revanche par son occupation permanente de la rue vit ce qui est identifié à une situation de rupture sociale. Les faqman sont définis comme : 
[des] enfants qui sont en rupture totale, partielle ou provisoire avec leurs familles et qui ont la rue pour lieu d'habitation. [Passant] entièrement leur temps, ou presque, dans la rue, ces " enfants de la rue " [...] sont des sujets qui échappent au contrôle de toute structure, même familiale, et qui vivent au jour le jour [...]. (UNICEF, 2007 : 26)

On peut ainsi suggérer que le fait que les faqman se soustraient à l'autorité des institutions sociales (famille, daara, école) participe à leur " étiquetage " (Becker, 1986) comme déviants, contrairement aux taalibe-mendiants plutôt perçus comme victimes et collant davantage à l'image d'une enfance à protéger.

La catégorie " enfant de la rue " s'est construite sur une conception occidentale voulant "que l'enfant soit avant tout ludique, innocent, authentique" (Stoecklin, $2000: 54)^{14}$. Cette représentation de l'enfance s'oppose à une présence de celui-ci dans un environnement perçu comme inadapté à son développement physique et psychique. $L^{\prime}$ « enfant de la rue » comme catégorie institutionnelle charrie des perceptions subjectives de l'enfant et de la rue qui font de l'enfant confronté à cet environnement un individu à secourir et à replacer dans des cadres et processus de socialisation considérés comme mieux adaptés. C'est ce qu'illustre l'action publique visant le retrait de la rue des taalibemendiants et la " modernisation " des daara, de même que les "projets de retrait et de réinsertion » initiés par des ONG locales. Considérer la diversité des catégories de la rue permet de voir que ces pratiques standardisées ne sont pas applicables à tous les profils. Prenons par exemple celui du faqman travailleur migrant. Obtenir la sortie de la rue de ce profil sans une alternative qui lui permette d'entrevoir la réalisation de l'objectif qui a motivé son départ vers la rue s'avère laborieux.

L. Salmon-Marchat (2004) a considéré le sens subjectif que les deux notions enfant et rue pouvaient avoir selon les contextes. Elle interroge la signification relative de la notion d'enfant. Sur le terrain déjà, son application peut être problématique dans la mesure où certains individus concernés sont juridiquement majeurs d'après la législation de leur pays. Le cas des faqman, où des adolescents âgés de 14 à 19 ans environ constituent la majorité de cette population, confirme cette observation. M. Champy (2015) pose un argument similaire lorsqu'elle préfère l'usage du terme bakoroman à celui d' « enfant de la rue » dans son étude du phénomène au Burkina Faso. Les longues années que nombre de ces jeunes passent dans la rue font qu'ils dépassent largement l'âge de la majorité (18 ans au Sénégal) qui circonscrit juridiquement la période de l'enfance. Ce critère de l'âge peut parfois être un facteur disqualifiant des pratiques de prise en charge, dans la mesure où celles-ci sont élaborées à destination des " enfants ". En tenant donc compte du caractère relatif des deux notions, rue et enfant, on parvient à une vision qui ne se résume plus à un problème social prenant racine dans un processus de déstructuration socio-économique qui engendre maltraitances, violences et vulnérabilités. Conception qui participe à tourner les regards vers les catégories qui paraissent les plus fragiles.

\footnotetext{
${ }^{14}$ M. Guidetti, S. Lallemand et M.-F. Morel (2002), dans une approche pluridisciplinaire de la notion d'enfance dans l'espace et dans le temps, ont montré que cette conception de l'enfant innocent et sujet de droits n'a pas toujours prévalu dans le monde occidental (européen).
} 
En prenant comme point de départ, que ce soit pour l'intervention ou la compréhension, des catégories empiriques (faqman, taalibe) plutôt que les représentations construites à partir de perceptions normatives, l'on constate que ces acteurs ne sont pas passifs face aux contraintes et pressions de divers ordres. R. AduayiDiop (2010) a étudié le travail des adolescentes sur les marchés de Dakar. Elle montre qu'une situation analysée à l'aune des catégories institutionnelles, comme un état de danger, de risques, de contraintes, d'entrave au développement et à l'épanouissement, peut être vue comme une stratégie de survie participant au processus de socialisation et d'intégration sociale de ses acteurs, lorsqu'elle est abordée sur la base d'une approche émique. Sous cet angle on peut voir que des populations identifiées comme vulnérables ou déviantes adoptent des stratégies d'adaptation, de résistance et d'innovation, dans le contexte de pauvreté, de changement social et structurel qui caractérise le Sénégal.

Outre l'avantage que cela représente dans le cadre de l'intervention, le décloisonnement de la catégorie " enfant de la rue " précise la nature de l'objet. II réduit les présupposés et dévoile la diversité des trajectoires et des objectifs qui motivent les individus désignés sous cette notion, ainsi que l'illustre l'exemple des faqman. Les définitions usuelles de l' " enfant de la rue " comme enfant en rupture ou comme enfant victime, expriment un point de vue de la société sur la catégorie à laquelle elles s'appliquent. Elles illustrent davantage les représentations d'un groupe social dominant qu'elles ne rendent vraiment compte d'une réalité. Comme l'exprime P. Gaboriau (2004 : 114) à propos des " sans-logis" en France, "rechercher une définition précise revient simplement [...] à dire la place occupée [ou attribuée] dans le champ social ». L'expression " enfant de la rue » dans son usage courant doit se rapporter à un problème social aux dimensions multiples plutôt qu'à une population homogène dont les individus partageraient des caractéristiques communes. Elle intègre dans les faits un nombre indéfini d'enfants et de jeunes qui partagent un contact ponctuel ou permanent avec la rue, mais se différencient par les motivations et les circonstances à l'origine de leur présence dans la rue, ainsi que par le rapport évolutif qu'ils construisent et entretiennent avec cet environnement.

Les limites de l'usage des catégories institutionnelles, qui identifient des états de vulnérabilité, ont été soulevées dans les études sur l'enfance dans le champ du développement. F. Hejoaka (2014) au Burkina Faso sur la catégorie OEV (orphelins et enfants vulnérables) remarquait que le ciblage établi dans le cadre des programmes de lutte contre le sida participe à une "concurrence des souffrances". En cherchant à normaliser des catégories construites, il aboutit à des formes d'exclusion. En effet dans ces pays dits en développement, ce qui est considéré par les acteurs de l'intervention comme vulnérabilité des enfants - en restant sur cet exemple du sida - peut provenir de causes indépendantes d'une infection ou d'une affection par la maladie. Les individus qui ne répondent pas aux critères établis par ces catégories sont de fait exclus des programmes sans que leur situation soit plus enviable que celle des bénéficiaires. D.M. Hoffman (2014) qui s'est intéressée à l'enfant dans le contexte de l'aide au développement à Haïti suggère dans ce sens une action davantage orientée sur les contextes à l'origine de la vulnérabilité, en lieu et place d'une centralité sur des sujets identifiés comme vulnérables. L'action 
publique qui se déploie autour des enfants dits vulnérables prendrait alors un tournant allant au-delà de l'accompagnement visant à "renforcer les capacités » qui doivent permettre de faire face à des conjonctures défavorables, pour inclure une action directe sur ces conjonctures. Ce ne sont plus alors les individus qui sont modelés de façon à s'insérer dans un système, mais des systèmes qui sont conçus de manière à s'adapter aux besoins des individus.

L'“ enfant de la rue ", qu'il soit taalibe, faqman, ou autre (enfants travaillant dans la rue, enfants mendiant aux côtés d'adultes, etc.), doit se penser dans la diversité de ses interactions avec les environnements qu'il fréquente: la rue, la famille, les centres d'accueil, etc. La compréhension de ce fait social n'est pas possible en dehors des dynamiques qui traversent les sociétés qui y sont confrontées (migrations, développement de nouvelles formes de sociabilités, transformations des structures sociales, etc.). Des dynamiques qui poussent aussi à réinterroger la place, le statut, les rôles que l'enfant occupe et joue désormais dans ces sociétés. La perspective relevant d'une conception supposée universelle de l'enfant crée un cloisonnement qu'il est nécessaire de dépasser. Pour ce faire, il convient aussi de saisir la rue en tant qu'univers de rencontres, d'interactions, d'expériences et d'opportunités, dévoilant l'émergence de sociabilités informelles (Lopez, 2009); et non plus seulement comme espace d'adversité. L'observation des catégories empiriques et une attention portée aux rationalités qui président à leur présence dans la rue ouvrent la voie à une meilleure appréhension des logiques qui sous-tendent les occupations et les usages divers que des jeunes font des espaces publics urbains.

\section{Bibliographie}

Aduayi-Diop R., 2010, Survivre à la pauvreté et à l'exclusion : le travail des adolescentes dans les marchés de Dakar, Paris, Karthala.

Aptekar L., 1994, "Street children in the developing world: A review of their condition ", Crosscultural research, vol. 28, $\mathrm{n}^{\circ} 3$, pp.195-224.

Becker H.S., 1986, Outsiders : études de sociologie de la déviance, Paris, Métaillé.

Champy M., 2015, " Ni enfants, ni adultes : une lecture comparative de la jeunesse (Burkina Faso) », Ateliers d'anthropologie [en ligne], $\mathrm{n}^{\circ} 42,14$ p. https://journals.openedition.org/ateliers/10024 (consulté le 06/11/2019).

Chehami J., 2016, "Les familles et les daara au Sénégal : entre facteurs macrosociaux, stratégies collectives et choix individuels ", Afrique contemporaine, $\mathrm{n}^{\circ} 257$, pp. 77-89.

Chehami J., 2013, Les " talibés » du Sénégal : une catégorie de la rue prise entre réseaux religieux et politiques humanitaires, thèse de doctorat en sociologie, IEP de Grenoble, $405 \mathrm{p}$.

Coloni M.-J., 1987, Sans toit ni frontière : les enfants de la rue, Paris, Fayard.

Collignon M., 1984, "La lutte des pouvoirs publics contre les "encombrements humains" à Dakar », Canadian Journal of African Studies, vol. 3, $\mathrm{n}^{\circ}$ 1, pp. 573-582.

Combier A., 1994, Les enfants de la rue en Mauritanie, Paris, L'Harmattan. 
Diack S., 2017, L'expérience des jeunes de la rue (faqman) à Dakar: entre marginalisation et reconnaissance sociale, thèse de doctorat en anthropologie, université Lyon II, $309 \mathrm{p}$.

Diop A.B., 2012, La société Wolof : tradition et changement, Paris, Karthala.

Diop M.-C., 1990, "L'administration sénégalaise et la gestion des 'fléaux sociaux' : I'héritage colonial ", Afrique et développement, vol. XV, n² 2, pp. 5-32.

Dramé F., 2010, Nàndité, enquêtes sur les enfants des rues à Dakar, Samu-Social Sénégal.

Dugrand C., 2013, " "Prendre la rue" : Les parcours citadins des Shégués de Kinshasa », Politique africaine, $\mathrm{n}^{\circ} 130$, pp. 189-212.

Ennew J., 1986, "Children of the street », New Internationalist, issue 164, October.

Faye O., thioub I., 2003, « Les marginaux et l'État à Dakar », Le mouvement social, n³, pp. 93-108.

Gaboriau P., 2004, "Mettre les questions à la question : travail de terrain et raisonnement sur les 'sans-logis' », Espaces et sociétés, n 116-117, pp. 111-123.

Guidetti M., Lallemand S., Morelle M.-F., 2002, Enfance d'ailleurs, d'hier et d'aujourd'hui : Approche comparative, Paris, Armand Colin.

Guillou A., 2009, "Au prisme du VIH : enquêter auprès des enfants des rues au Cambodge ", Anthropologie et sociétés, vol. 33, $\mathrm{n}^{\circ} 1$, pp. 101-122.

Hejoaka F., 2014, "La concurrence des souffrances. Genèse et usages électifs de la catégorie des orphelins et enfants vulnérables au temps du sida ", Autrepart, $n^{\circ} 72$, pp. 59-75.

Hoffman D.M., 2014, "Slaves and angels: the child as a developmental casualty in Haïti », Autrepart, $\mathrm{n}^{\circ}$ 72, pp. 95-109.

Hugon C., 2015, «Les sëriñ-daara et la réforme des écoles coraniques au Sénégal : Analyse de la fabrique d'une politique publique ", Politique africaine, $n^{\circ} 139$, pp. 83-99.

Human Rights Watch, 2017, Je vois encore des talibés mendier: Insuffisance du programme gouvernemental pour protéger les enfants talibés au Sénégal, rapport, $44 \mathrm{p}$.

Human Rights Watch, 2014, Exploitation sous prétexte d'éducation: Progrès mitigés de la lutte contre la mendicité forcée des enfants au Sénégal, rapport, $53 \mathrm{p}$.

Human Rights Watch, 2010, Sur le dos des enfants : Mendicité forcée et autres mauvais traitements à l'encontre des talibés au Sénégal, rapport, 20 p.

Lopez R.P., 2009, Vivre et survivre à Mexico : Enfants et jeunes de la rue, Paris, Karthala.

Lucchini R., 2001, "Carrière, identité et sortie de la rue : le cas de l'enfant de la rue ", Déviance et société, vol. $25, n^{\circ} 1$, pp. 75-97.

Lucchini R., 1998, "L'enfant de la rue : réalité complexe et discours réducteur ", Déviance et société, vol. $22, n^{\circ} 4$, pp. 347-366.

Lucchini R., 1996, Sociologie de la survie : l'enfant de la rue, Paris, PUF.

Lucchini R., 1993, Enfant de la rue : identité, sociabilité et drogue, Genève, Droz.

Salmon-Marchat L., 2004, Les enfants de la rue à Abidjan : une étude socio-anthropologique des éléments symptomatiques de la crise ivoirienne, Paris, L'Harmattan. 
Sané K., 2017, Les talibés mendiants, une catégorie d'enfants de la rue au Sénégal, thèse de doctorat, université Paris-Est, 587p.

Stoecklin D., 2000, Enfants des rues en Chine, Paris, Karthala.

Taracena E., 1995, "Enfants de la rue et enfants dans la rue à Mexico ", Revue lien social et politique, $\mathrm{n}^{\circ} 34$, pp. 101-107.

Thorsen D., Jacquemin M., 2015, "Temporalités, savoir-faire et modes d'action des enfants travailleurs migrants au sein de la parenté élargie en Afrique de l'Ouest ", Canadian Journal of African Studies / Revue canadienne des études africaines, vol. 49, n² 2, pp. 285-299.

UNICEF, 2007, Enfants mendiants dans la région de Dakar, rapport, Dakar, 87 p. 